\title{
Tecnochess. Una propuesta didáctica para trabajar las competencias lingüística, tecnológica y matemática mediante el juego del ajedrez y las TIC $^{1}$
}

\author{
Tecnochess. A Teaching proposal to work linguistic, technological and \\ mathematical competences with chess and ICT \\ Héctor García Millán \\ Universidad de Lleida \\ hgarciamillan@gmail.com \\ Ángel Blanch Plana \\ Universidad de Lleida \\ ablanch@pip.udl.cat
}

Recibido: 19/07/2016

Aceptado: $12 / 12 / 2016$

Publicado: 29/12/2016

\section{RESUMEN}

La inclusión de las competencias básicas del currículo de educación primaria requiere nuevos planteamientos en materia de diseño e implementación curricular. El presente artículo sugiere una propuesta de enseñanza innovadora para abordar las competencias lingüística, tecnológica y matemática (LTM) mediante el uso del ajedrez y las nuevas tecnologías en educación primaria. El ajedrez es un recurso didáctico útil en entornos educativos con el objetivo de desarrollar competencias curriculares. Este trabajo explora esta vía, proponiendo materiales y actividades centradas en la instrucción de las LTM a través del juego del ajedrez y las nuevas tecnologías.

\section{PALABRAS CLAVE}

Competencias en educación primaria; ajedrez; nuevas tecnologías.

\section{ABSTRACT}

The inclusion of the core competencies of the primary education curriculum requires new approaches in curriculum design and implementation. This article suggests an innovative teaching approach to address linguistic, technological and mathematical (LTM) skills through the use of chess and new technologies in primary education. Chess is a useful didactic resource in educational settings aiming at developing curricular competencies. The current study explores this possibility by proposing teaching materials and activities that focus on the instruction of LTM through the game of chess and new technologies.

\section{KEYWORDS}

Competencies in primary education; chess; new technologies.

\section{CITA RECOMENDADA}

García, H. y Blanch, A. (2016). Tecnochess. Una propuesta didáctica para trabajar las competencias lingüísticas, tecnológica y matemática mediante el juego del ajedrez y las TIC . RIITE. Revista Interuniversitaria de Investigación en Tecnología Educativa, 1, 39-51. Doi: http://dx.doi.org/10.6018/riite/2016/263991

\footnotetext{
${ }^{1}$ Este estudio se enmarca en el programa del Máster Oficial Interuniversitario en Tecnología Educativa: elearning y gestión del conocimiento (http://www.tecnologiaeducativa.eu/)
} 
Principales aportaciones del artículo y futuras líneas de investigación:

- Innovación educativa en educación primaria: ajedrez, TIC y competencias básicas.

- Desarrollo de las competencias LTM a través de nuevas metodologías.

- El ajedrez en las escuelas como método de adquisición de nuevas competencias.

- Nuevas metodologías de enseñanza a través del juego del ajedrez.

\section{INTRODUCCIÓN}

El uso de las nuevas tecnologías ha generado cambios constantes en la sociedad actual que afectan de manera directa e indirecta al mundo educativo. Estos cambios han marcado el quehacer de las instituciones educativas a la hora de adaptarse a los nuevos tiempos que corren integrando las nuevas tecnologías en la práctica educativa. Como consecuencia de ello los centros no solo han tenido que incorporar recursos tecnológicos, sino que también implica un trabajo extra acumulativo, como por ejemplo rediseñar los modelos pedagógicos tradicionales para adaptarlos a las nuevas exigencias, con cambios en la metodología docente, formación, organización escolar, los roles del profesorado y el alumnado, los formatos y diseño de contenidos de aprendizaje, los recursos o las propuestas de actividades (Expósito y Manzano, 2012).

A pesar de ello, es necesario destacar los esfuerzos por introducir las TIC en el mundo educativo ya que generan cambios significativos en el aprendizaje, en la motivación e interés, el rendimiento académico y en el desarrollo de nuevas competencias (Ricoy, Feliz y Sevillano, 2010). Competencias como la lingüística, la del ámbito digital o la matemática idóneas para el desarrollo de propuestas didácticas innovadoras.

El hecho de relacionar el ajedrez con las TIC supone un reto en el desarrollo de propuestas didácticas innovadoras ya que hasta el momento son muy pocos los estudios realizados al respecto (Aciego, García, y Betancort, 2016). Estos factores, conjuntamente con la posibilidad de añadir TECNOCHESS a la práctica educativa, nos motivan a trabajar en la mejora de la propuesta. Por otra parte, durante el desarrollo de la propuesta se realizó un análisis selectivo de los contenidos, los métodos de enseñanza-aprendizaje y los materiales a utilizar con el fin de que el alumnado pudiera desarrollar las competencias LTM de una manera motivadora.

\subsection{Ajedrez y educación}

Algunos autores realzan el valor educativo del juego del ajedrez como materia de aprendizaje y desarrollo de estructuras mentales lógico-matemáticas (Piaget, 1978). Del mismo modo, el ajedrez favorece la atención, la concentración, la paciencia, el cálculo, el análisis, la comunicación, así como el desarrollo de la creatividad, la intuición, la memoria, el razonamiento matemático, la organización y la lectura (Kovacic, 1996). De la misma manera, el ajedrez estimula la capacidad de analizar y deducir a partir de principios generales, aprender a tomar decisiones difíciles y resolver problemas de una manera flexible y autónoma (Dauvergne, 2000; Gliga y Flesner, 2014).

En Texas, Estados Unidos, se realizó un estudio interesante tomando como grupo experimental a un grupo de estudiantes de educación primaria de ciclo medio, entre los cursos de tercero y quinto, que participaban en un club de ajedrez perteneciente a la escuela a la que 
formaban parte. Dicho grupo experimental mostró una mejora muy significativa en las áreas de lectura y matemáticas, ya que puntuaron el doble en relación con el grupo control (niños que no eran jugadores de ajedrez) en el Texas Assessment of Academic Skills (TAAS) (Liptrap, 1998).

Asimismo durante la década de los 80 se realizó en Venezuela otro estudio donde el Ministerio de Desarrollo de Inteligencia formó a cien mil docentes para que enseñaran habilidades del pensamiento a una muestra inicial de 4.266 estudiantes de segundo ciclo. Este estudio se apeló con el nombre de "Proyecto: Aprendiendo a pensar" en el que se demostró cómo el ajedrez puede ayudar al desarrollo de la inteligencia de los alumnos y dicho desarrollo puede ser evaluado con la Escala de Inteligencia para Niños de Wechsler (WISC). Durante el transcurso del experimento, los estudiantes manifestaron un crecimiento significativo en el cociente intelectual después de haber estudiado sistemáticamente el juego del ajedrez durante menos de un año. De esta manera se concluyó que "el ajedrez enseñado metodológicamente es un sistema de incentivo suficiente para acelerar el aumento de cociente intelectual en niños en edades de educación primaria en ambos sexos y en todos los niveles socio-económicos" (Ferguson, 2001).

Para justificar la forma en que se puede mejorar el desarrollo de la competencia lingüística a partir de programas ajedrecísticos, encontramos un estudio realizado en Nueva York que involucró más de 100 escuelas y 3000 alumnos mostrando una mejora significativa en las materias de inglés y matemáticas (Margulies, 2007). Otro estudio describe la organización de dos clases de cada una de las cinco escuelas en dos grupos, uno experimental y otro control. Sólo los grupos experimentales recibieron instrucción del juego del ajedrez. Posteriormente se observó como todos los grupos experimentales obtuvieron puntuaciones mucho más elevadas en lectura que los grupos control (Margulies, 2007). Resultados similares también se han observado recientemente en relación a habilidades matemáticas (Sala, Gorini, y Pravettoni, 2015; Trinchero y Sala, 2016).

\subsection{Ajedrez y nuevas tecnologías}

La escuela tiene que preparar al alumnado para la vida real y proporcionar herramientas y aplicaciones digitales, pues son una parte indisociable de nuestra vida. La comprensión del mundo que nos rodea está condicionada, por cómo se muestra este mundo y cómo nos comunicamos. Por tanto, los medios digitales y el dominio de las herramientas digitales son decisivos para que el alumnado sea competente en el mundo tecnológico en el que vivimos (Palm, 1990).

Actualmente nos encontramos en un momento de la historia de permanentes cambios constantes, no estamos delante de una simple cuestión de moda ni de exigencia consumista, sino que la escuela no puede permanecer al margen de la tecnología digital. El dominio de la tecnología por parte del alumnado es superficial por mucho que se hable de nativos digitales y requiere de un proceso de enseñanza - aprendizaje donde hay que ordenar y seleccionar los aprendizajes más relevantes para que los alumnos lleguen a ser ciudadanos competentes. Por lo tanto, es un reto para las escuelas actuales proporcionar las herramientas y aplicaciones de la tecnología digital al alcance de todo el alumnado sin renunciar a su función educativa en todos los aspectos, y por ello el profesorado no puede permanecer al margen de unas competencias digitales que son un hecho ineludible del mundo educativo actual.

En relación a lo antedicho, en 1990 se realizó en varias escuelas de Estados Unidos una investigación que relacionaba varias aplicaciones informáticas con el uso del ajedrez en las aulas experimentando una mejora en la práctica académica de los alumnos. Concluyendo que, después de cuatro años de haber establecido el programa de ajedrez, se comprobó cómo este 
juego introduce en los niños/as un gran sentimiento de autoconfianza y autoestima, desarrolla el pensamiento racional, mejora las habilidades cognitivas y comunicacionales, a la vez que aumenta el rendimiento académico (Palm, 1990).

Cinco años más tarde se realizó otro estudio relacionado con los ordenadores y el ajedrez en una escuela de Bradford, Estados Unidos, mostrando con la utilización de un instrumento de medición cómo los puntajes de los test mejoraban en un 173\% en el alumnado implicado en las clases de ajedrez. El resultado fue comparado con el resultado de solo un $4,56 \%$ para los alumnos que participaban en otros programas de "enriquecimiento" que incluían: resolución de problemas futuros, resolución de problemas con la utilización de computadoras, estudio independiente, escritura creativa, entre otros. La evaluación de valoración derivó un resultado en el que el ajedrez favorecía habilidades relacionadas con el pensamiento crítico en mayor medida que otros métodos de desarrollo (Ferguson, 1995).

Finalmente y haciendo referencia a las nuevas tecnologías, éstas permiten relacionar varias prácticas educativas con diferentes recursos ya que nos permiten desarrollar nuevos materiales didácticos, de carácter electrónico, que utilizan diferentes soportes. Soportes informativos como por ejemplo Internet, que aporta un lenguaje propio, unos códigos específicos orientados a generar otras formas de comunicación y otros entornos de aprendizaje colaborativos que sin duda pueden relacionarse con nuevas metodologías como la que estamos planteando en este innovador proyecto.

\subsection{Competencias curriculares}

La inclusión de las competencias básicas en el currículo de primaria exige la adopción de una nueva perspectiva para su diseño y desarrollo. Su incorporación no ha de entenderse como si fuera un elemento más, sino que precisamente el cambio conceptual y metodológico viene de la mano de la consideración de las competencias como el eje en torno al cual han de girar todos y cada uno de los elementos curriculares (Sierra, 2012).

Este enfoque ha de llevar a planificar las programaciones desde una nueva perspectiva tanto por parte de los centros educativos como por parte de los docentes. El aprendizaje basado en competencias ha de constituir una continuidad en la que se dispongan el resto de los elementos del currículo. Dicho de otra manera, las competencias básicas deberán complementarse en todo momento con los objetivos de aprendizaje, los contenidos y actividades, y la evaluación, mediante metodologías que permitan al alumnado ser activo y partícipe de su aprendizaje concediendo a la vez un impulso significativo en el desarrollo de sus propias competencias (Sierra, 2012).

Por otra parte, el currículo de educación primaria apuesta por una orientación de la enseñanza obligatoria hacia el desarrollo de las competencias básicas. Dicho currículo define las competencias básicas como un conjunto complejo de conocimientos, habilidades, actitudes, valores, emociones y motivaciones que cada individuo o grupo pone en acción en un contexto concreto para hacer frente a las demandas peculiares de cada situación.

Se consideran competencias fundamentales aquellas que son imprescindibles para que las personas puedan hacer frente a las exigencias de los diferentes contextos de la vida como ciudadanos. Es decir, aquellas competencias que son importantes para muchos ámbitos de la vida y que contribuirán a una vida satisfactoria y al buen funcionamiento de la comunidad.

El currículo establece además que para que el alumnado adquiera eficazmente las competencias básicas será necesario que los docentes diseñen actividades de enseñanza y aprendizaje que permitan desarrollar una o varias competencias a la vez. Este enfoque exige al 
profesorado reflexionar sobre cómo desarrollar las clases y cómo plantear las prácticas educativas para que el alumnado adquiera dichas competencias.

\subsection{Las competencias LTM}

El planteamiento de TECNOCHESS se centra en las competencias LTM (Lingüística, Tecnológica, y Matemática) incluyendo así diferentes elementos del currículo y, por consiguiente atendiendo a las exigencias que se plantean desde el mismo (Figura 1). Además, con el aporte de las nuevas tecnologías se adapta a la funcionalidad espacio-tiempo ya que permite un aprendizaje sin limitaciones asistenciales ni temporales, provocando así una nueva visión educativa que permite al alumnado aprender y madurar a su propio ritmo.

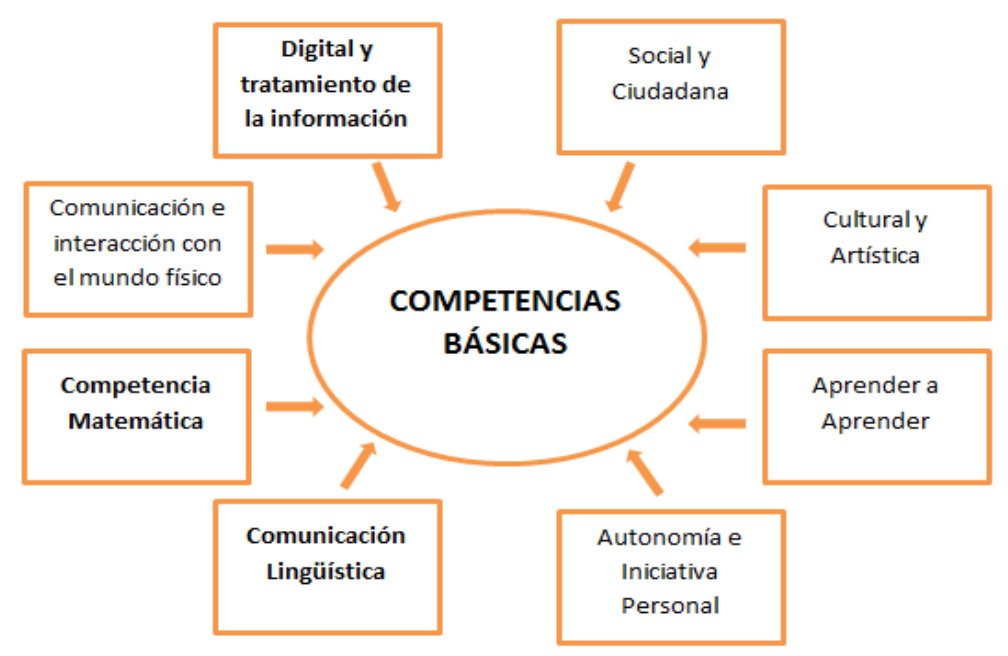

Figura 1. Relación de las competencias básicas en educación primaria.

\section{METODOLOGÍA DIDÁCTICA}

El objetivo general de TECNOCHESS se centra principalmente en adaptar los materiales que utilizamos a las competencias básicas que se establecen en el currículo de primaria. De esta manera nos proponemos diseñar una propuesta de intervención educativa para el desarrollo de las competencias LTM, a partir de una metodología basada en las TIC y el juego del ajedrez.

Asimismo, los objetivos específicos de la propuesta que se pretenden alcanzar con el desarrollo del proyecto son: a) proponer la implementación y utilización de aplicaciones, plataformas y otras herramientas TIC centradas en el ajedrez para mejorar las competencias LTM; b) formalizar una propuesta de intervención didáctica para trabajar las competencias LTM a partir del ajedrez y las TIC.

La propuesta didáctica que presentamos se diseñó bajo una metodología de aprendizaje innovadora mediante el juego del ajedrez y las nuevas tecnologías, pretendiendo ser una alternativa en el proceso de enseñanza-aprendizaje y el desarrollo de las competencias básicas de educación primaria. Esta metodología supuso tener en cuenta, entre otros aspectos, el contexto educativo, la definición de las actividades que permitieran alcanzar objetivos de aprendizaje, la planificación de las sesiones enfocadas a una línea constructivista del aprendizaje y, finalmente, la estructuración del método de evaluación. 
Teniendo en cuenta estos aspectos, el diseño de la propuesta se organizó en 3 fases relacionadas entre sí y enfocadas en la adquisición de las competencias LTM (Figura 2).

Asimismo, las fases de la propuesta se apoyaron en el uso de diferentes recursos y herramientas tecnológicas que permitieran al alumnado desarrollar las sesiones bajo un enfoque colaborativo para adquirir los aspectos competenciales establecidos en el currículum.

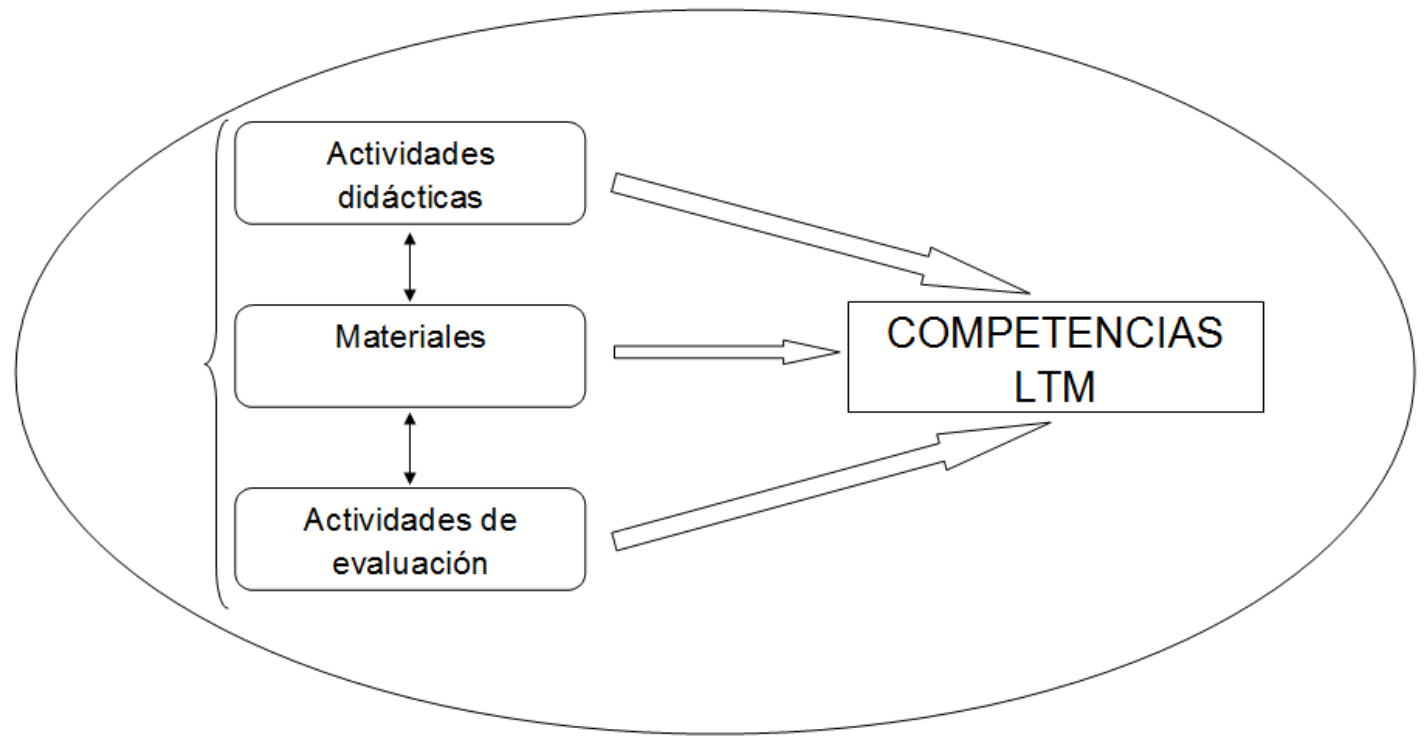

Figura 2. Fases en el proceso de creación de la propuesta didáctica.

\section{Fase 1: Diseño de las actividades didácticas}

En la primera fase de la propuesta se definieron una serie de actividades individuales y colectivas que combinaban el uso de varios recursos físicos (tablero de ajedrez, fichas, etc.) con diversas aplicaciones y herramientas tecnológicas como ordenadores, tabletas electrónicas o las PDI (pizarras digitales interactivas) entre otras. El hecho de utilizar estos recursos nos permitió desarrollar de manera gradual algunas de las competencias tecnológicas establecidas en el currículo. Estas actividades tenían la finalidad de iniciar al alumnado en el aprendizaje básico del ajedrez y posibilitar la adquisición de las competencias LTM.

Por otro lado, la propuesta se diseñó dirigida al ciclo inicial (6-8 años) y ciclo medio (8-10 años) de educación primaria ya que, como hemos mencionado anteriormente, se trata de una programación inicial con actividades para alumnos que tienen un conocimiento nulo o muy básico del ajedrez.

Por otra parte, durante el proceso de realización de las actividades didácticas se seleccionaron y organizaron los contenidos de manera precisa para facilitar una adquisición progresiva de contenidos. Asimismo, se trabajó en el diseño de actividades basadas en el aprendizaje a través del juego, introduciendo de esta manera actividades variadas, dinámicas y motivadoras.

Fase 2: Diseño y organización de materiales 
De manera paralela a la realización de las actividades didácticas y las actividades de evaluación se fueron seleccionando de manera precisa los materiales más idóneos para cada una de las actividades. En esta línea se seleccionó el material teniendo en cuenta el contenido de aprendizaje, el estado madurativo del alumnado al cuál iba dirigido, la metodología a utilizar en cada una de las sesiones y las características específicas de los materiales a utilizar.

Por otra parte se recurrió a materiales que facilitaran la adquisición de los objetivos de aprendizaje. En esta medida se seleccionaron materiales económicos y de fácil adquisición para que la propuesta pudiera ser desarrollada en cualquier centro escolar. Para ello investigamos a fondo la web con la intención de proporcionar los mejores materiales para el proceso de enseñanza-aprendizaje y el desarrollo de las competencias LTM. También se consideró la posibilidad de que las TIC fallaran, proporcionando otros materiales físicos para que el desarrollo de las sesiones no se viera perjudicado por posibles errores tecnológicos que pudieran surgir durante el desarrollo de las sesiones.

\section{Fase 3. Diseño de las actividades de evaluación}

En el diseño de las actividades de evaluación se tuvo en cuenta la progresión de las actividades didácticas anteriormente realizadas. Es decir, cada sesión de actividades didácticas contiene una o dos actividades de evaluación deviniendo así en una evaluación continua. También se valoró la evaluación autónoma e individual de cada alumno, y la progresividad de las actividades en el tiempo de aprendizaje del juego del ajedrez puesto que consideramos que es imprescindible para el desarrollo de las competencias LTM.

Por otra parte, en este nivel de la propuesta se relacionaron los objetivos de evaluación con las competencias básicas y específicas del programa. Si bien, no se creyó oportuno trabajar cada una de las competencias de manera íntegra, pues consideramos que las competencias se deben ir adquiriendo progresivamente durante los tres ciclos de primaria, y por tanto será más productivo e interesante profundizar en éstas a medida que vaya evolucionando el aprendizaje y la edad del alumno. A continuación se muestra un ejemplo de una de las sesiones de evaluación de la propuesta didáctica (Figura 3).

\begin{tabular}{|c|c|c|}
\hline & $\begin{array}{c}\text { ACTIVIDAD DE } \\
\text { EVALUACIÓN UNIDAD } 1\end{array}$ \\
\hline \multicolumn{2}{|c|}{$\begin{array}{l}\text { UNIDAD DIDÁCTICA: Unidad 1. El tablero: las casillas, las líneas y las piezas. } \\
\text { BLOC DE CONTENIDOS: Las piezas y su posición } 2 \\
\text { OBJETIVO DIDÁCTICO: Resolver un problema reconociendo la posición de las casillas } \\
\text { del tablero de ajedrez. } \\
\text { COMPETENCIA: L (C4, C5, C8) M (C1, C4, C6, C7, C8, C9) T (C1, C2, C6, C9, C10) } \\
\text { FASE }\end{array}$} & MATERIAL \\
\hline Iniciación & $\begin{array}{l}\text { Actividad 6. Escribe en el tablero las letras en mayúsculas y podrás leer los } \\
\text { diferentes personajes del ajedrez. } \\
\text { Esta actividad se puede realizar colectivamente en el aula a través de la } \\
\text { pizarra digital o también individualmente a través de las tabletas } \\
\text { electrónicas. } \\
\text { Para realizar la actividad será imprescindible que el docente haya creado y } \\
\text { colocado en cada tableta electrónica un archivo.docx donde habrá creado } \\
\text { un tablero de ajedrez editable para que los alumnos puedan escribir las } \\
\text { letras que se les pide en la actividad y descubrir así los diferentes } \\
\text { personajes que existen en el juego del ajedrez. } \\
\text { Para realizar la actividad los alumnos se tendrán que guiar de la } \\
\text { nomenclatura ajedrecística enseñada en sesiones anteriores. } \\
\text { Al finalizar la actividad cada alumno deberá guardar su trabajo en el menú } \\
\text { Archivo/Guardar como/Actividad_nombredelalumno.docx } \\
\text { Para el docente esta actividad realizada individualmente puede servirle de } \\
\text { evaluación sin necesidad de realizar ningún examen al finalizar cada } \\
\text { unidad. }\end{array}$ & $\begin{array}{l}\text {-PDI (Pizarra Digital } \\
\text { Interactiva) o proyector } \\
\text { digital conectado a un } \\
\text { ordenador. } \\
\text { - Tableta electrónica, } \\
\text { ordenador. } \\
\text { - Apps: Microsoft Office } \\
\text { Word o similar. }\end{array}$ \\
\hline
\end{tabular}

Figura 3. Ejemplo de la sesión de evaluación 


\section{ACTIVIDADES}

Las actividades elaboradas en TECNOCHESS se organizaron en dos tipos: las actividades didácticas con las que el alumnado va adquiriendo conocimientos del juego del ajedrez y las TIC, y las actividades de evaluación donde se involucra al alumnado en un trabajo autónomo que le permita adquirir varias habilidades o dimensiones reflejadas en las competencias LTM del currículo de primaria. A continuación se muestran algunas actividades donde se trabajan las competencias LTM y las dimensiones descritas anteriormente.

\subsection{El puzle del caballo}

Esta actividad trabaja varias dimensiones de las competencias LTM mediante un juego lúdico del caballo del ajedrez. Se trata de una actividad individual en la que el alumnado deberá recorrer y pintar con su caballo todos los cuadros blancos que se encuentre en el tablero. Por supuesto, para pintar dichos cuadros deberemos desplazarnos con el movimiento en forma de "L" característico del caballo de ajedrez. Antes de iniciar la actividad el docente deberá explicar en la PDI o el proyector digital cómo se puede acceder al juego a través del link: http://www.troyis.com/?lang=es.

El juego es una plataforma que se desarrolla por niveles empezando por el nivel 1 de máxima facilidad y complicándose a medida que vamos avanzando de nivel. A mayor nivel alcanzado mayor puntuación obtendremos.

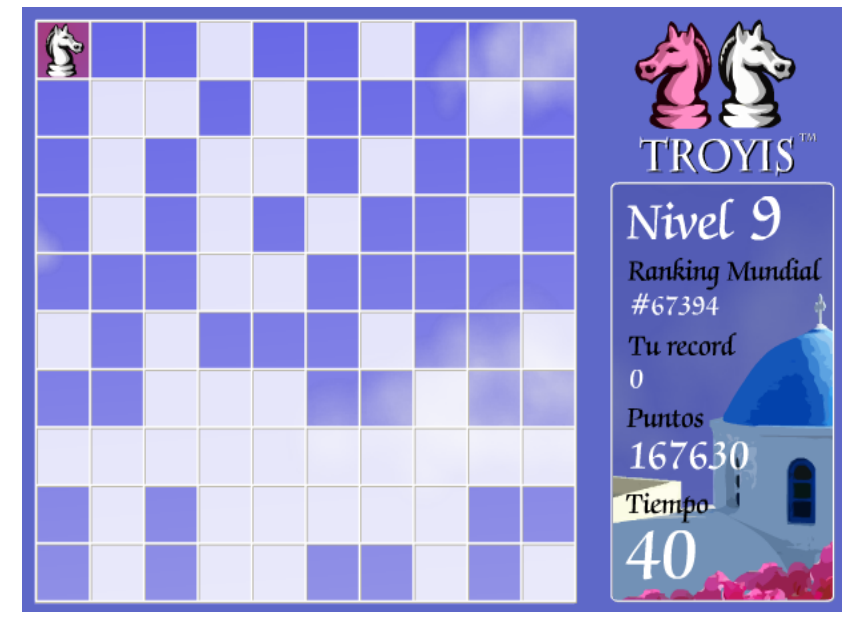

Figura 4. El puzle del caballo.

\subsection{Crazychess}

Teniendo en cuenta que el movimiento del caballo en el tablero es el más complicado del juego, hemos considerado oportuno mostrar una segunda actividad lúdica sobre esta pieza que permite interiorizar la captura de piezas con el caballo y sus movimientos de una manera más amena.

Crazychess es una actividad similar a la anterior pero en esta ocasión en vez de tener que recorrer los diferentes cuadros, nuestro caballo blanco deberá evitar que los peones negros nos capturen y que tampoco coronen en octava fila puesto que si no iremos perdiendo vida en la partida. 

mediante el juego del ajedrez y las TIC

Esta actividad tiene la ventaja de que se puede realizar en el aula de inglés para aprender algunas palabras básicas anglosajonas ya que no está disponible en castellano.

Una característica interesante de este juego es que los alumnos podrán elegir el nivel de juego recomendando a los más noveles el nivel más básico. Los niveles van de mayor a menor facilidad en este orden: novato, amateur, pro y gran máster. Algunos de estos niveles necesitan de méritos conseguidos en niveles inferiores como por ejemplo conseguir una medalla de plata para poder avanzar al nivel gran máster.

El objetivo de este juego es capturar el mayor número de peones y extras (monedas, peones, caballos, etc.) describiendo el correcto movimiento del caballo y siempre evitando que los peones coronen en octava fila o nos capturen, pues eso nos debilitará a la hora de conseguir mayor puntuación.

Esta actividad es interesante desarrollarla individualmente con las tabletas electrónicas si es posible y si no es así también se podrá desarrollar con otras herramientas TIC como ordenadores o móviles. http://www.minijuegostop.com.mx/items/estrategia/0/112 el-caballo-deajedrezl

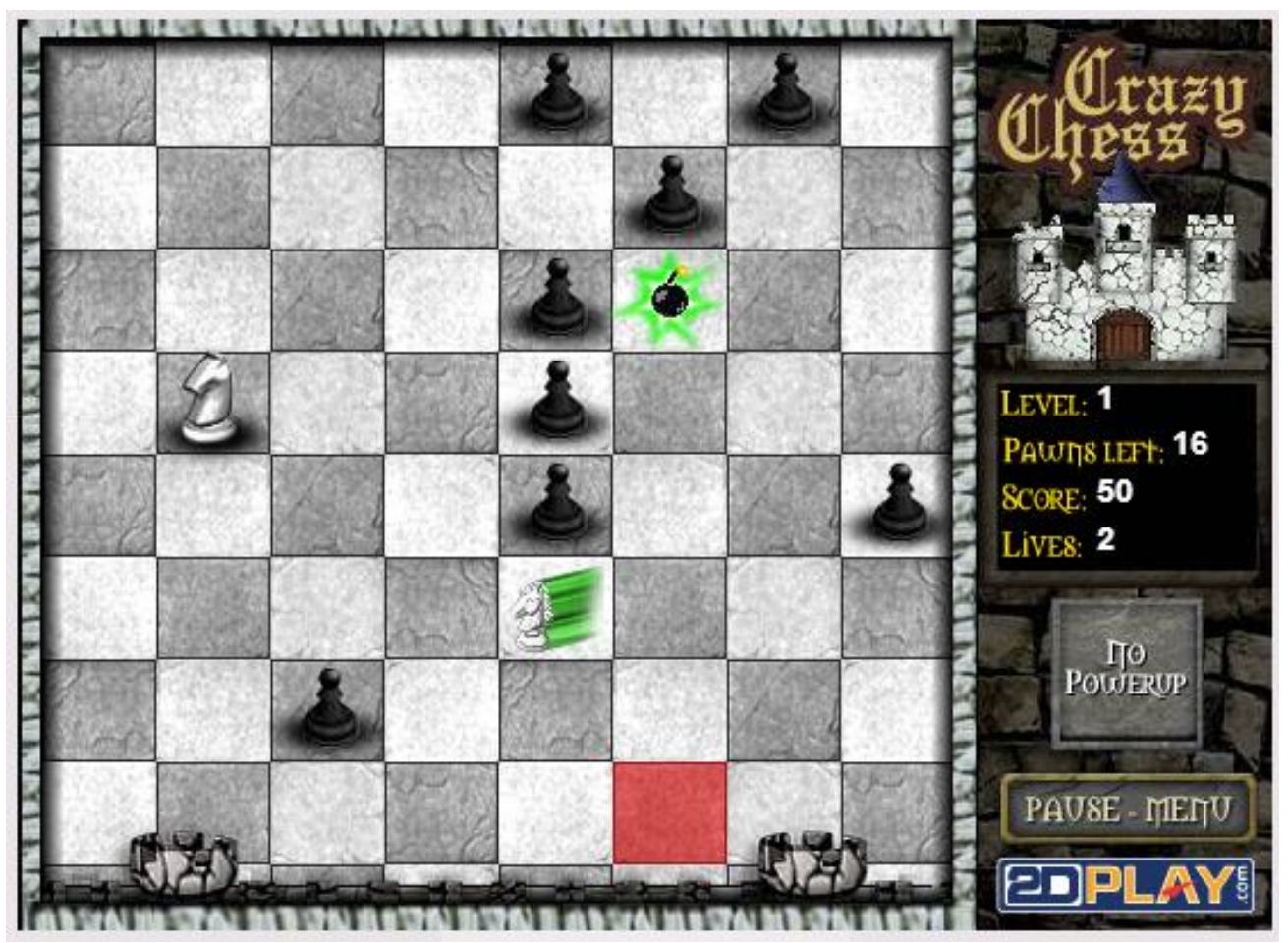

Figura 5. Crazychess

\subsection{Ajedrez por parejas}

Esta actividad consiste en jugar una partida de ajedrez por parejas. Para ello es imprescindible el uso de tabletas electrónicas u ordenadores puesto que nuestro propósito es realizar esta actividad a través de las nuevas tecnologías.

Una vez realizados los grupos de 2 contra 2 que organizará en un principio el docente, se les facilitará una dirección web (https://ajedrez.chess.com/analysis-board-editor) donde podrán jugar la partida.

El juego consiste en jugar por turnos. Los participantes enumerarán en un equipo de dos miembros, jugador número 1 y jugador número 2, contra los oponentes enumerados de igual 
manera. Los turnos de juego empezarán por el equipo blanco dónde el jugador número uno moverá una pieza, lo que el oponente número 1 "negro" también moverá una pieza, después moverá el jugador blanco número 2 para después mover el jugador negro número 2 y así sucesivamente.

Las reglas del "ajedrez por parejas" son las mismas que las del ajedrez convencional, pero con la diferencia de que en este juego hay que respetar los turnos de los jugadores y tener totalmente prohibido hablar durante la partida, sancionando con la partida perdida si se produce. Por tanto, las señas, signos y palabras quedan prohibidas en este juego.

Con este tipo de actividad se pretende fomentar la visión de juego del compañero y de los oponentes en elaboración de las jugadas, también despertar el análisis de juego en la jugada inmediata, es decir, fomentar las típicas preguntas del: ¿por qué mueve esta pieza? ¿Qué puedo hacer yo? Pero no olvidemos del componente lúdico del juego para despertar el interés hacia el ajedrez. Con esta actividad también se trabajan otros objetivos como potenciar el silencio y la concentración.

Para que el juego se desarrolle correctamente el docente tendrá que ser tajante y remarcar mucho las normas de la actividad. También sería interesante hacer una pequeña competición con el grupo clase por parejas. De esta manera se motivaría a los alumnos a tomarse en serio más la actividad, aunque sí es cierto que la actividad necesitaría varias sesiones y puede que no sea motivante para todos los alumnos.

Por último, la ventaja que tiene realizar la actividad a través de la plataforma sobre el tablero físico es que esta web no permite volver atrás después de realizar una jugada. Por tanto, deberemos remarcar que han de pensar muy bien las jugadas antes de realizarlas.

Como variante adicional de esta actividad podremos poner el reloj digital que descargamos en las sesiones anteriores o combinarlo con un reloj físico para jugar al ajedrez que sin duda harán la actividad más atractiva.

En el caso que no tuviéramos Internet en el aula o las nuevas tecnologías fallaran siempre podríamos realizar la actividad con el tablero físico de ajedrez y sus respectivas piezas.

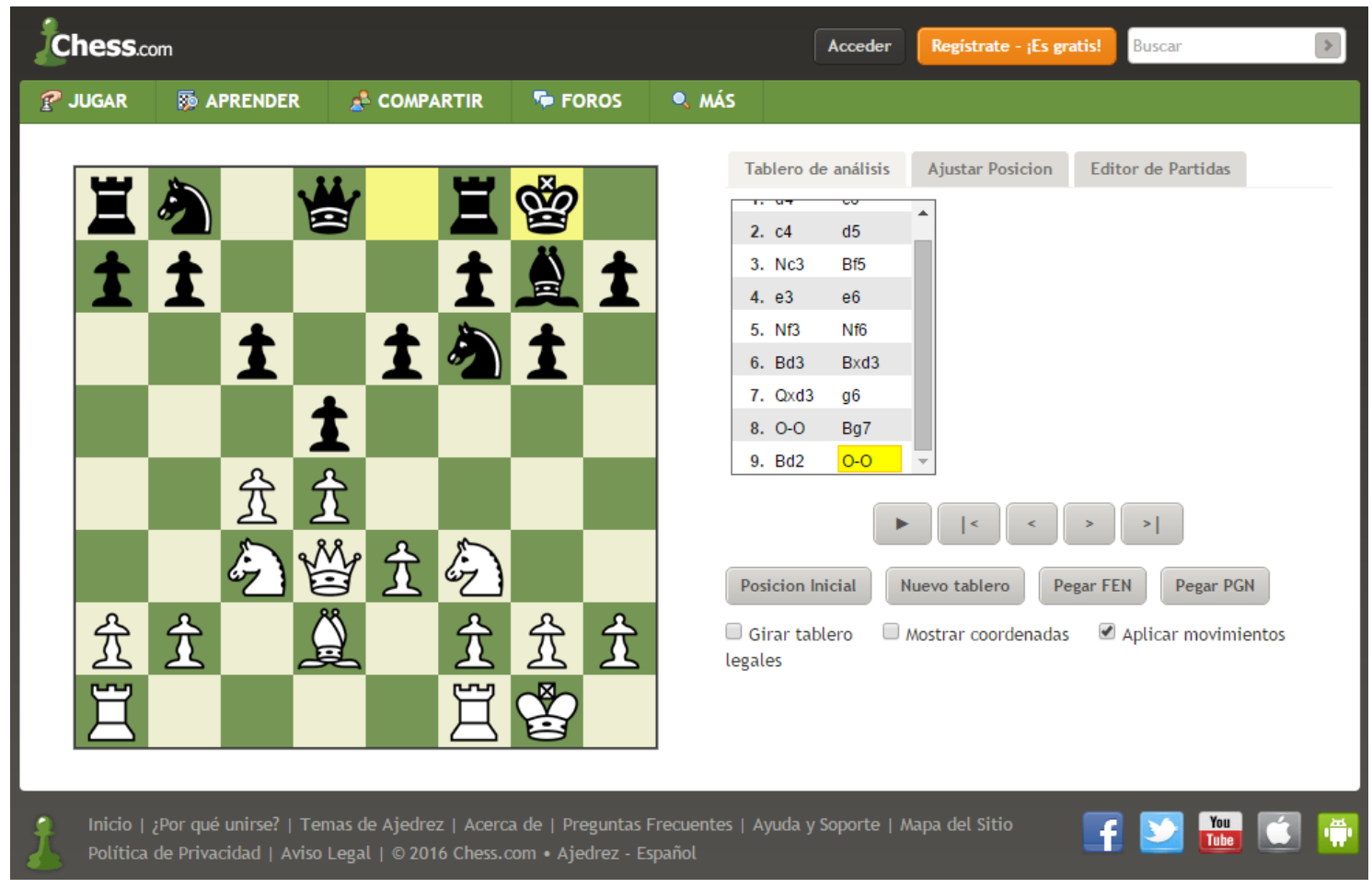


Figura 6. Plataforma donde practicar el juego del ajedrez por parejas

\section{CONCLUSIONES}

Vincular las nuevas tecnologías y el ajedrez en una propuesta didáctica como un instrumento innovador en el desarrollo de las competencias LTM no siempre genera una innovación sustantiva de los métodos didácticos y de las prácticas docentes. TECNOCHESS pretende utilizar las TIC en un proyecto de trabajo en el que los alumnos puedan resolver situaciones problemáticas en base al aprendizaje del ajedrez.

Por otra parte, desde TECNOCHESS destacamos el planteamiento como un eje innovador para la adquisición de las competencias LTM a través de un aprendizaje lúdico y dinámico que pretende mostrar otros métodos de enseñanza más motivadores para el alumnado. El uso de recursos lúdico-pedagógicos aprovecha la disposición innata del estudiante a los juegos, introduciendo de esta manera una actividad llena de motivación, sacando lo mejor del alumno, y apostando por el desarrollo de competencias en un ambiente tranquilo y dinámico que incite al aprendizaje de las competencias LTM.

Es evidente que muchos docentes actuales desconocen los conocimientos fundamentales del ajedrez, siendo éstos imprescindibles para poder desarrollar esta propuesta. Desde esta perspectiva nos gustaría animar desde estas líneas a aquellos docentes con espíritu de superación en el aprendizaje del ajedrez, puesto que los beneficios que conlleva su práctica son sustanciales.

Por otra parte, reconocemos que hacer del ajedrez un recurso integrador para satisfacer las demandas educativas del currículum no es una tarea sencilla. Sabemos que además de su valor educativo también ha de contribuir al desarrollo de competencias específicas que permitan adquirir habilidades, aplicar conocimientos y técnicas estudiadas en situaciones de aula, que favorezcan el trabajo autónomo y cooperativo del alumnado.

En definitiva, el programa de intervención educativa que realizamos para el desarrollo de las competencias LTM pretende convertirse en un recurso curricular útil que permita introducir, orientar y estructurar la práctica de dichas competencias en el alumnado. Por tanto, tomando como base la labor realizada, cada docente deberá adaptar y adecuar esta propuesta según el contexto y las características del alumnado. Resulta indiscutible que esta manera de pensar y actuar representa un revulsivo para la práctica educativa actual. La libertad de poder introducir propuestas innovadoras ligada a la construcción de un currículum adaptable y global en la vida de las aulas, puede convertirse en una solución a las pautas y hábitos de enseñanza tradicionales de nuestras escuelas que sin duda, nos abre un camino de esperanza hacia una nueva manera de ver la educación de hoy y del mañana.

\section{RECONOCIMIENTOS}


Este trabajo se ha realizado con la colaboración de la Cátedra de Ajedrez, Educación y Desarrollo Cognitivo, Universitat de Lleida.

\section{REFERENCIAS BIBLIOGRÁFICAS}

Aciego, R., García, L. y Betancort, M. (2016). Efectos del método de entrenamiento en ajedrez, entrenamiento táctico versus formación integral, en las competencias cognitivas y sociopersonales de los escolares. Universitas Psychologica, 15, pp. 165-176.

Expósito, J. y Manzano, B. (2012). New digital learning models in educational process. Acta Humanitá, 4, año 2012. Faculty of Humanities: Universitiy of Žilina.

Ferguson, R. C. (2001, diciembre). Solving Academic Problems. Ponencia presentada en la Conferencia Internacional de Ajedrez KoltanowskiChess. Dallas (USA).

Gliga, F., y Flesner, P.I. (2014). Cognitive benefits of chess training in novice children. Procedia - Social and Behavioral Sciences, 116, 962-967.

Kovacic, D.M. (1996). Ajedrez en las escuelas. Una buena movida. Psiencia. Revista latinoamericana de ciencia psicológica, 4 (1), pp. 29-41.

Krogius, N. V. (1972). La psicología en ajedrez. Barcelona: Editorial Martínez Roca.

Liptrap, J. M. (1998). Chess and standard test scores. Chess Life, 41-43.

Margulies, S. (2007). The Effect of Chess on Reading Scores: District Nine Chess Program Second Year Report. New York: The American ChessFundation.

Palm, C. (1990). Chess improves academic performance. United States Chess Federation Scholastic Department.

Piaget, J. (1978). La equilibración de las estructuras cognitivas. Problema central del desarrollo. Madrid: Siglo XXI.

Ricoy, M. C., Feliz, T. y Sevillano, M. L. (2010). Competencias para la utilización de las herramientas digitales en la Sociedad de la Información. Educación XXI, 13 (1), 199- 219.

Sala, G., Gorini, A., y Pravettoni, G. (2015). Mathematical problem-solving abilities and chess: An experimental study on young pupils. Sage Open, 5.

Sierra, B. (2012). La programación por competencias básicas: hacia un cambio metodológico interdisciplinar. Revista Complutense de Educación, 24, (1), 165-184.

Trinchero, R., y Sala, G. (2016). Chess training and mathematical problem solving: The role of teaching heuristics in transfer of learning. Eurasia Journal of Mathematics, Science \& Technology Education, 12, pp. 655-668.

\section{INFORMACIÓN SOBRE LOS AUTORES}

\section{Héctor García Millán}

Universidad de Lleida

Graduado en educación primaria y educación física de la Universidad de Lleida y máster en Tecnología Educativa: E-learning y gestión del conocimiento. Líneas de investigación: ajedrez y nuevas tecnologías en educación, métodos y técnicas de investigación en psicología y educación, desplegamiento curricular del ajedrez en la escuela. 


\section{Ángel Blanch Plana \\ Universidad de Lleida}

Profesor agregado en el Departamento de Psicología de la Universidad de Lleida. Doctor en psicopedagogía, ingeniero técnico en informática de gestión, y educador social.

Líneas de investigación: diferencias individuales, rendimiento intelectual, salud laboral, métodos y técnicas de investigación en psicología y educación. Editor asociado de Personality and Individual Differences. Revisor de revistas científicas en psicología y educación.

\section{(cc) EY-NC}

Los textos publicados en esta revista están sujetos a una licencia de Reconocimiento 4.0 España de Creative Commons. Puede copiarlos, distribuirlos, comunicarlos públicamente y hacer obras derivadas siempre que reconozca los créditos de las obras (autoría, nombre de la revista, institución editora) de la manera especificada por los autores o por la revista. La licencia completa se puede consultar en:Licencia Creative Commons Atribución-NoComercial-Compartir por igual 4.0 Internacional. 